\title{
Functionalization at Will of Rim-Differentiated Pillar[5]arenes
}

\author{
Paul Demay-Drouhard, ${ }^{\dagger,}, \nabla_{0}$ Ke Du, ${ }^{\dagger, \nabla_{0}}$ Kushal Samanta, ${ }^{\dagger, t, \nabla_{0}}$ Xintong Wan, ${ }^{\dagger}$ (이 Weiwei Yang,

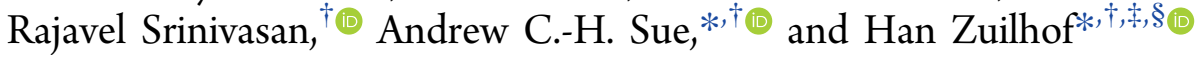

$\dagger$ Institute for Molecular Design and Synthesis, School of Pharmaceutical Science \& Technology, Tianjin University, 92 Weijin Road, Nankai District, Tianjin, 300072, People's Republic of China

${ }^{\ddagger}$ Laboratory of Organic Chemistry, Wageningen University, Stippeneng 4, 6703 WE Wageningen, The Netherlands

${ }^{\S}$ Department of Chemical and Materials Engineering, King Abdulaziz University, 21589 Jeddah, Saudi Arabia

\section{Supporting Information}

ABSTRACT: The development of an efficient synthetic route toward rim-differentiated $C_{5}$-symmetric pillar $[5]$ arenes $(\mathrm{P}[5] \mathrm{s})$, whose two rims are decorated with different chemical functionalities, opens up successive transformations of this macrocyclic scaffold. This paper describes a gram-scale synthesis of a $C_{5}$-symmetric penta-hydroxy $\mathrm{P}[5]$ precursor, and a range of highly efficient reactions that allow functionalizing either rim at will via, e.g., sulfur(VI) fluoride exchange (SuFEx) reactions,
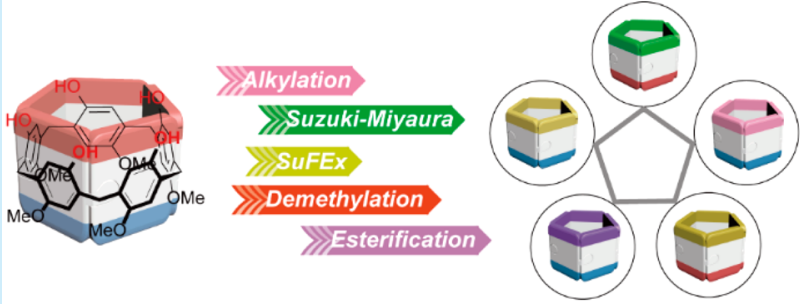
esterifications, or Suzuki-Miyaura coupling. Afterward, $\mathrm{BBr}_{3}$ demethylation activates another rim for similar functionalizations.

$\mathrm{T}$ he last decades have witnessed significant developments of supramolecular chemistry, ${ }^{1}$ largely inspired by Nature's highly effective approaches to combine both covalent and noncovalent interactions. An important class of supramolecules are macrocyclic compounds, such as cyclodextrins, ${ }^{2}$ calixarenes, ${ }^{3,4}$ and, more recently, pillarenes, ${ }^{5,6}$ especially those of five repeating aromatic units (pillar[5] arenes or $\mathrm{P}[5] \mathrm{s}$; see Figure 1). The latter class has characteristic features that they can

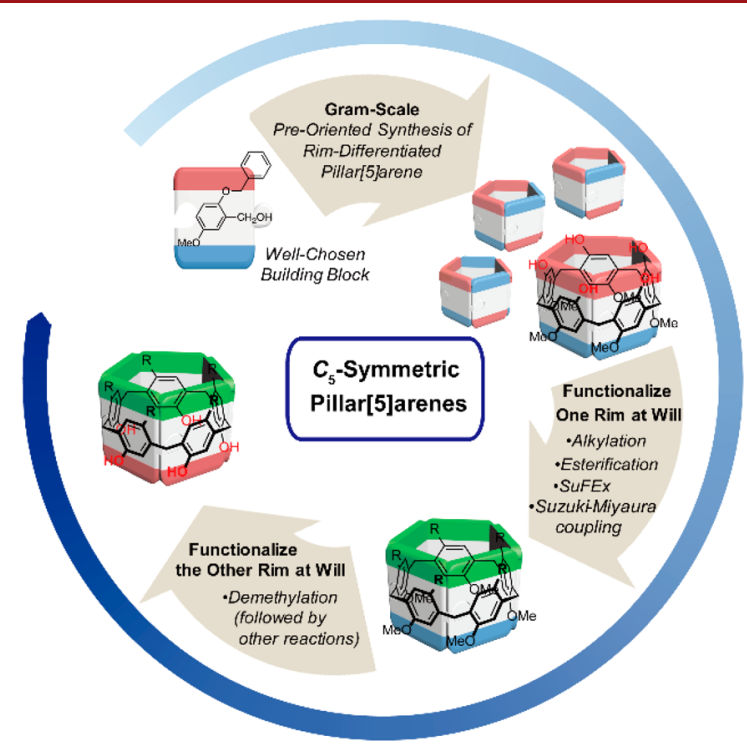

Figure 1. Functionalization at will on both sides of $C_{5}$-symmetric rimdifferentiated pillar[5]arenes leads to a series of transformations of this macrocyclic scaffold. routinely be synthesized in gram-scale quantities and are easy to obtain via various routes. The pillarlike shape, in combination with their electron-rich nature, ${ }^{7,8}$ has made these attractive platforms for separation ${ }^{9-15}$ applications, and building blocks for complex supramolecular architectures. ${ }^{16-19}$

Most common $\mathrm{P}[5] \mathrm{s}$ are per-functionalized, with 10 identical substituents, which are appealing for their high symmetry and facile synthesis. ${ }^{20,21}$ However, a wealth of additional structural complexity and functionality can be added by alternative functionalization schemes, ${ }^{22}$ including monosubstitution, ${ }^{23,24} \mathrm{~A} 1 / \mathrm{A} 2$ disubstitution, ${ }^{25-27}$ phenylene substitution, $^{28,29}$ methylene bridge functionalization, ${ }^{28,29}$ and rim differentiation..$^{30,31}$ The latter approach, which led to tiara$\mathrm{P}[5] \mathrm{s}$, have received relatively less attention, since there is no straightforward pathway capable of converting easily available $D_{5}$-symmetric per-functionalized $\mathrm{P}[5] \mathrm{s}$ into their corresponding $C_{5}$-symmetric rim-differentiated ones. Cyclization of asymmetrically substituted 1,4-dialkoxybenzene monomers is feasible, but generates the expected $C_{5}$-symmetric isomer in low selectivity and poor yields $(\leq 5 \%) .^{32-36}$ Recently, this statistical process was recently greatly improved by employing the so-called preoriented strategy ${ }^{37,38}$ starting from asymmetrically substituted 2,5-dialkoxybenzyl alcohols, thereby optimizing the syntheses of $C_{5}$-symmetric isomers with selectivity higher than $50 \%$ and isolated yields up to $20 \%$. This development paved the way for more widespread applications and novel chemistries of rim-differentiated $\mathrm{P}[5]$ platforms, which have already been used as amphiphilic self-assemblies bearing hydrophilic and hydrophobic groups on opposite

Received: March 30, 2019 
Scheme 1. A Divergent Synthetic Route, Starting from the Gram-Scale Synthesis of a Common Penta-Hydroxy RimDifferentiated $\mathrm{P}[5]$ Precursor $(\mathrm{OH})_{5}-\mathrm{P}[5]^{a}$
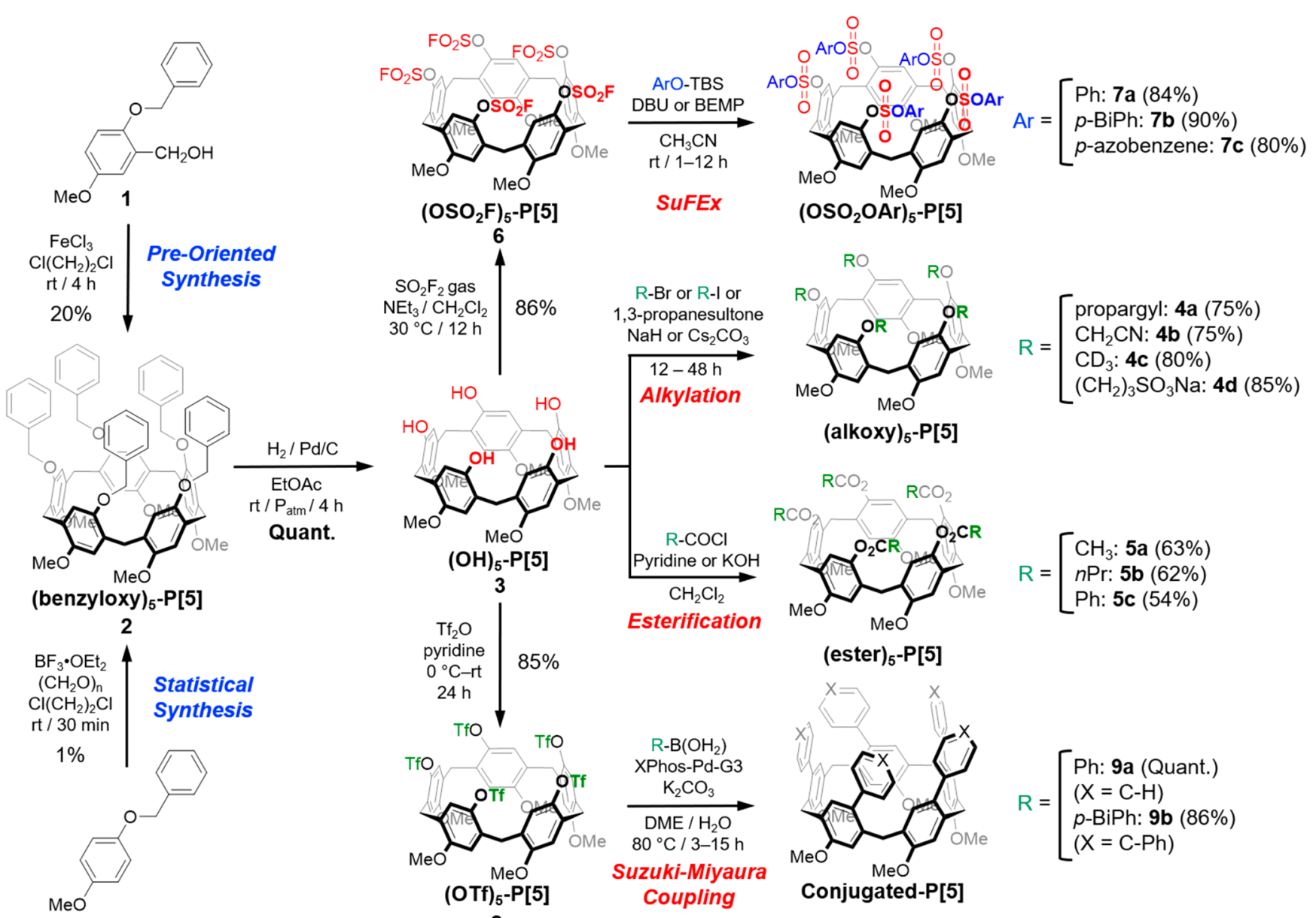

$(\text { alkoxy) })_{5}-\mathrm{P}[5]$
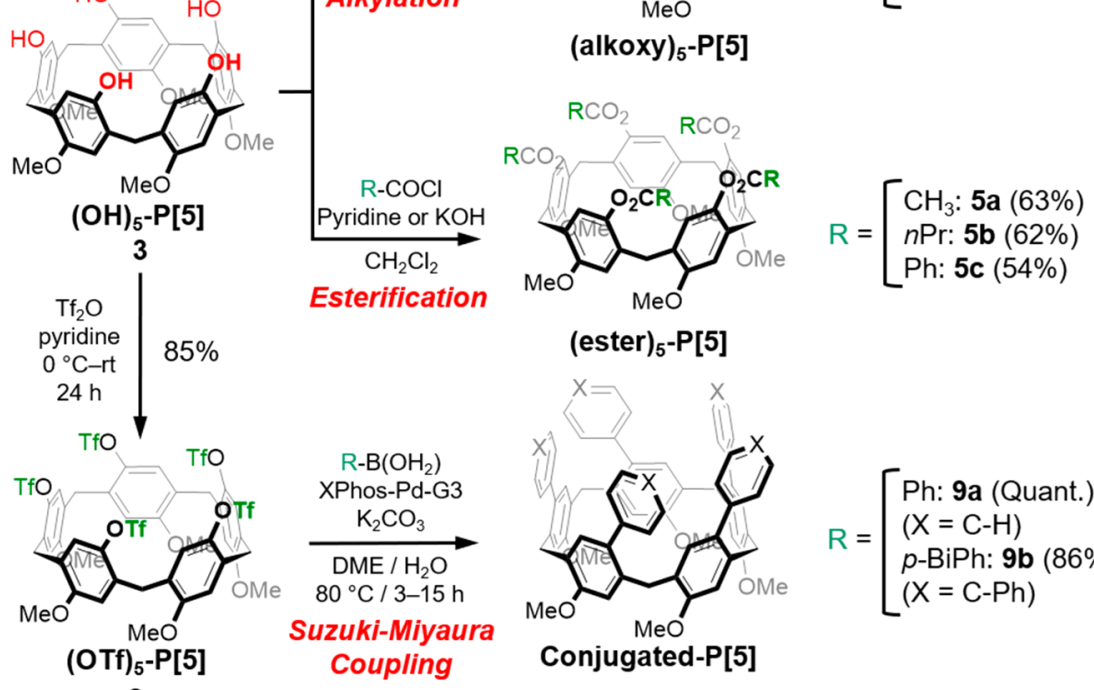

$$
\mathrm{R}=\left[\begin{array}{l}
\text { Ph: 9a (Quant.) } \\
(\mathrm{X}=\mathrm{C}-\mathrm{H}) \\
\text { p-BiPh: } 9 \mathrm{~b}(86 \%) \\
(\mathrm{X}=\mathrm{C}-\mathrm{Ph})
\end{array}\right.
$$

${ }^{a_{\text {This }}}$ route leads to a variety of $C_{5}$-symmetric $\mathrm{P}[5]$ derivatives through alkylation, esterification and SuFEx reactions.

rims $^{39-43}$ and as promising candidates for multivalent surface grafting. ${ }^{44,45}$ Although the cyclization step has been made more efficient, this preoriented protocol requires the synthesis of the corresponding dialkoxybenzyl alcohol monomer for each target compound, and the subsequent purification by column chromatography can be nontrivial. These limitations clearly hamper the development of this method. A universal rimdifferentiated $\mathrm{P}[5]$ building block that can be produced on a large scale and freely functionalized at either side is thus highly desired (Figure 1).

In the current paper, we propose an alternative divergent synthetic route (see Scheme 1) to overcome these limitations and widely open the door toward rim-differentiated $\mathrm{P}[5] \mathrm{s}$ that can be functionalized at will. Key in this route is the efficient, gram-scale synthesis of a common intermediate, namely, $(\mathrm{OH})_{5}-\mathrm{P}[5]$ (the five methoxy groups on the other rim are omitted hereafter, for the sake of clarity), which is a rimdifferentiated $\mathrm{P}[5]$ equipped with hydroxyl moieties on one side. As it happens, this $(\mathbf{O H})_{5}-\mathrm{P}[5]$ was recently reported by $\mathrm{Al}$-Azemi et al. $^{46}$ from the corresponding (OBn) $5-\mathbf{P}[5]$. However, in their report, this benzyl derivative was obtained via the statistical method from 4-benzyloxyanisole, followed by hydrogenation. The laborious separation of different constitutional isomers led to a very low isolated yield $(<1 \%)$ for the rim-differentiated $(\mathbf{O H})_{5}-\mathbf{P}[5]$, which certainly precludes any large-scale synthesis of multiple derivatives starting from this compound.

As a first step, we thus used our preoriented protocol with (2-(benzyloxy)-5-methoxyphenyl)methanol, which was easily synthesized in two steps from commercially available reagents ( $82 \%$ overall yield; typical scale of product $=13 \mathrm{~g}$ ). The pentacyclization reaction thereof yielded the expected rim-differentiated (OBn) $)_{5}-\mathbf{P}[5]$ in a much improved $20 \%$ yield in gramscale batches ( $1.2 \mathrm{~g}$ product per reaction; see the Supporting Information for detailed synthetic procedures) after straightforward column chromatography, followed by quick recrystallization (both using $n$-hexane/EtOAc). (OBn) $)_{5} \mathbf{P}[5]$ was subsequently hydrogenated ${ }^{27,46}\left(\mathrm{H}_{2}\right.$ over $\left.\mathrm{Pd} / \mathrm{C}\right)$, quantitatively yielding the corresponding penta-hydroxy $(\mathrm{OH})_{5}-\mathrm{P}[5]$.

Single-crystal samples of air-unstable $(\mathrm{OH})_{5}-\mathrm{P}[5]$ were obtained by vapor-vapor diffusion ( $n$-hexane/EtOAc) under Ar protection. X-ray crystallography revealed (Figure $2 \mathrm{a}$ ) that $(\mathrm{OH})_{5}-\mathrm{P}[5]$ adopts a distorted pentagonal conformation similar to per-hydroxylated $\mathrm{P}[5],{ }^{47}$ in which two aromatic units are flipped, relative to the other three, as a result of the three intramolecular hydrogen bonds formed between $\mathrm{OH}$ and $\mathrm{OCH}_{3}$ groups on neighboring phenylene rings $([\mathrm{O} \cdots \mathrm{O}]$ distances of 2.81, 2.81, and $2.67 \AA$; see Figure 2a, presented later in this work). In addition, two intermolecular $\mathrm{O}-\mathrm{H} \cdots \mathrm{O} \mathrm{H}$ bonds can be found between different macrocycles, and 
between the $\mathrm{P}[5]$ macrocycle and its EtOAc guest in the cavity ([O… $]$ distances of 2.82 and $2.81 \AA$ ).

For subsequent syntheses, $(\mathbf{O H})_{5}-\mathrm{P}[5]$ was typically used immediately after hydrogenation, to avoid air oxidation (see Scheme 1). As earlier attempts, $(\mathbf{O H})_{5}-\mathbf{P}[5]$ was realkylated with propargyl bromide and $\mathrm{NaH}$ at $60{ }^{\circ} \mathrm{C}$ to afford pure

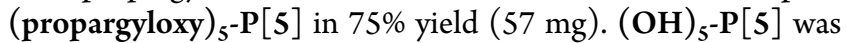
also reacted with bromoacetonitrile to generate $\left(\mathrm{OCH}_{2} \mathbf{C N}\right)_{5^{-}}$ $\mathbf{P}[\mathbf{5}]$, also in $75 \%$ yield, whose nitrile moiety could easily be reduced to the corresponding amine later on. While this divergent synthetic route seems to afford merely rimdifferentiated products already obtainable via our preoriented route, this procedure is advantageous: the involvement of a $C_{5^{-}}$ symmetric $\mathrm{P}[5]$ that can be synthesized and purified with high efficiency as the common precursor leads to isomerically pure final products in the subsequent reactions. In addition, this route uniquely enables the synthesis of $C_{5}$-symmetric $\mathrm{P}[5] \mathrm{s}$ whose isolation from the corresponding constitutional isomers is nontrivial (e.g., (propargyloxy) $5-\mathbf{P}[\mathbf{5}])^{38}$ or simply (near-)impossible. A clear case of minute differences, down to only isotopic substitution between the two rims, clearly demonstrates the strength of this approach. We achieved the synthesis of $\mathrm{CH}_{3}$ vs $\mathrm{CD}_{3}$ rim-differentiated $\left(\mathrm{OCD}_{3}\right)_{5}-\mathrm{P}[\mathbf{5}]$, an isotopologue of per-methylated $\mathrm{P}[5]$, by reacting $\mathrm{CD}_{3} \mathrm{I}$ with $(\mathrm{OH})_{5}-\mathrm{P}[5]$ ( $80 \%$ yield). Another noteworthy, rim-differentiated and highly water-soluble pentasulfonate $\mathbf{P}[\mathbf{5}]((\mathbf{O}-$ $\left.\left.\left(\mathrm{CH}_{2}\right)_{3} \mathrm{SO}_{3} \mathrm{Na}\right)_{5}-\mathrm{P}[5]\right)$ was obtained by reacting $(\mathrm{OH})_{5}-\mathrm{P}[5]$ with 1,3-propane sultone ( $85 \%$ yield; $150 \mathrm{mg}$ ).

Moreover, this route provides extra functional group tolerance for moieties that are incompatible with the Friedel-Crafts cyclization conditions toward $\mathrm{P}[5]$, such as esters. In the current approach, esterification by reacting rimdifferentiated $(\mathbf{O H})_{5}-\mathbf{P}[\mathbf{5}]$ with acyl chlorides is rather easy, providing access to (OCOMe $)_{5}-\mathbf{P}[\mathbf{5}]$ and (OCOPr) $-\mathbf{P}[\mathbf{5}]$ in typically $>60 \%$ yields $(65-80 \mathrm{mg}$ scale $)$ at room temperature (rt). Harsher conditions were required for the less-reactive benzoyl chloride $\left(\mathrm{KOH}\right.$ in refluxing $\mathrm{CH}_{2} \mathrm{Cl}_{2} / \mathrm{H}_{2} \mathrm{O}$ ), but the expected (OCOPh) $)_{5}-\mathrm{P}[5]$ could be isolated in 54\% yield.

While alkylation and esterification reactions generally result in fair to very good yields, highly efficient chemistry that converts five reactive handles on rim-differentiated $\mathrm{P}[5]$ simultaneously is still highly desired. An alternative approach to functionalization of the $-\mathrm{OH}$ moieties of $(\mathrm{OH})_{5}-\mathrm{P}[5]$ is the sulfur(VI) fluoride exchange (SuFEx) reaction. ${ }^{48}$ This SuFEx click chemistry has proven to be especially useful to obtain high yields in constrained environments, ${ }^{49}$ as present near the rim of $\mathrm{P}[5]$. $(\mathrm{OH})_{5}-\mathrm{P}[5]$ was cleanly reacted with sulfuryl fluoride gas in the presence of base to obtain the corresponding fluorosulfate. This $\left(\mathrm{OSO}_{2} \mathbf{F}\right)_{5}-\mathrm{P}[5]$ was allowed to react with a range of aromatic tert-butyldimethylsilyl (TBS) ethers to give rim-differentiated $\mathrm{P}[5] \mathrm{s}$ with penta- $\mathrm{OSO}_{2} \mathrm{OPh}$, $\mathrm{OSO}_{2} \mathrm{O} p$ - $\mathrm{BiPh}$, and photoswitchable ${ }^{50,51} \mathrm{OSO}_{2} \mathrm{O} p$-azobenzene (see the Supporting Information for UV-vis characterization) moieties on one rim, respectively. All reactions proceeded in good to excellent isolated yields after simple recrystallization or precipitation and $\mathrm{MeOH}$ washing (see Scheme 1; the amount of isolated product is typically $100 \mathrm{mg}$ ). Given the wide, and still rapidly expanding, scope and high efficacy of SuFEx reactions, we would argue that this methodology provides significant potential to a broad range of $\mathrm{P}[5] \mathrm{s}$ with different functionalization patterns and other types of macrocycles and supramolecules.
All previous routes still vary around the theme of functionalization of the oxygen atom. However, the functionality of $\mathrm{P}[5]$ s can be altered drastically if the $\mathrm{O}$ atom itself could be replaced and, in this way, changing the electron density of the macrocyclic scaffold (see Scheme 1). In order to achieve this, and directly connect aryl groups on the $\mathrm{P}[5]$ core, $(\mathrm{OH})_{5}-\mathrm{P}[\mathbf{5}]$ was converted to the corresponding penta-triflate, (OTf) ${ }_{5}-\mathbf{P}[\mathbf{5}]$, in $85 \%$ yield $(330 \mathrm{mg}$ ). This compound was engaged in Suzuki-Miyaura couplings to generate $(\mathbf{P h})_{5}-\mathbf{P}[\mathbf{5}]$ and $(p-\mathbf{B i P h})_{5}-\mathbf{P}[\mathbf{5}]$ in excellent isolated yields $(86 \%-100 \%$ on a $50-150 \mathrm{mg}$ scale) after straightforward column chromatography with $n$-hexane/ethyl acetate. The use of $\mathrm{XPhos}$ coupled to a third-generation Buchwald precatalyst (XPhos-Pd-G3) ${ }^{52}$ proved essential to the success of these couplings, since conventional Pd-based catalysts, e.g., Pd$\left(\mathrm{PPh}_{3}\right)_{4}$, failed to give complete conversion. The compounds shown possess an extended aromatic cavity, where biphenyl and even terphenyl moieties are the 5-fold repeating units. Since they are devoid of oxygen atoms on one rim, this should greatly modify the electronic properties ${ }^{53}$ of the cage.

The last series of reactions we undertook involved the deprotection of the methoxy groups on the other rim, and, in that way, provided access to full and independent control of the functionalities present on both rims (see Scheme 2).

Scheme 2. Activating the Other Rim for Further Functionalization: Demethylation of Various RimDifferentiated $\mathbf{P}[\mathbf{5}] \mathbf{s}$

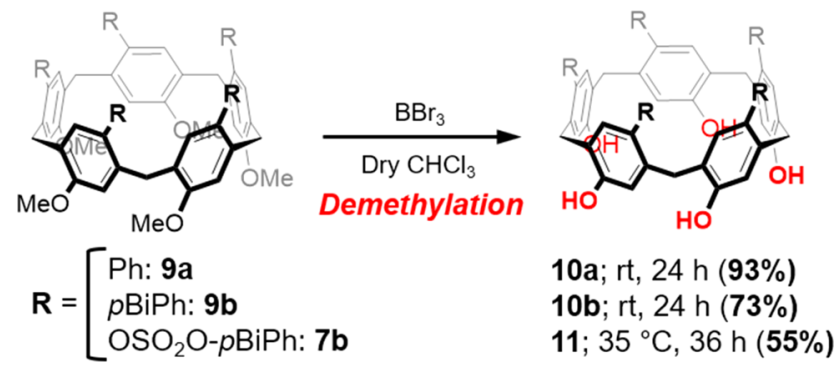

Common $\mathrm{P}[5] \mathrm{s}$ are decorated with multiple alkoxy groups on both rims that cannot be removed selectively, and this grossly limits the design of novel $\mathrm{P}[5] \mathrm{s}$. In our work, robust $\mathrm{C}-\mathrm{C}$ and $\mathrm{OSO}_{2} \mathrm{O}$ linkages are effected through SuzukiMiyaura coupling and SuFEx reactions on one rim of $\mathrm{P}$ [5] first, thus opening up the other rim for further reactions via $\mathrm{BBr}_{3}$ demethylation. As typical examples, conjugated $(\mathbf{P h})_{5}-\mathbf{P}[\mathbf{5}]$ and $(p-\mathbf{B i P h})_{5}-\mathbf{P}[5]$ were reacted with $\mathrm{BBr}_{3}$ in dry $\mathrm{CHCl}_{3}$, to afford the corresponding penta-hydroxy compounds in $70 \%-$ $90 \%$ yields. Analogously, the five $\mathrm{OCH}_{3}$ at the lower rim of $\left(\mathrm{OSO}_{2} \mathrm{O} p-\mathrm{BiPh}\right)_{5}-\mathbf{P}[\mathbf{5}]$ were also cleanly demethylated, yielding corresponding penta-OH compounds in $55 \%$ isolated yields. All these compounds are readily available for further transformations via synthetic strategies described above, thus obtaining the desired freedom of functionalization on both rims at will.

The structures of $(\mathrm{OH})_{5}-\mathrm{P}[5],\left(\mathrm{OCH}_{2} \mathrm{CN}\right)_{5}-\mathrm{P}[5],(\mathrm{Ph})_{5}-$ $\mathrm{P}[5],(\mathrm{Ph})_{5}(\mathrm{OH})_{5}-\mathrm{P}[5], \quad(p-\mathrm{BiPh})_{5}-\mathrm{P}[5], \quad(p-\mathrm{BiPh})_{5}(\mathrm{OH})_{5}-$ $\mathrm{P}[5],\left(\mathrm{OSO}_{2} \mathrm{OPh}\right)_{5}-\mathrm{P}[5]$, and $\left(\mathrm{OSO}_{2} \mathrm{O} p \text {-azobenzene }\right)_{5}-\mathrm{P}[5]$ were all confirmed via X-ray crystallography (Figure 2 ). The expected regular pentagonal cavity was observed for each $\mathrm{P}[5]$, except for $(\mathbf{O H})_{5}-\mathbf{P}[5]$, as discussed earlier. 
(a)

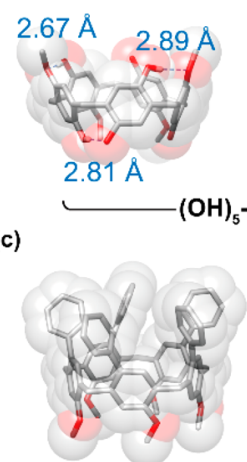

$(\mathrm{Ph})_{5}-\mathrm{P}[5]$

(f)

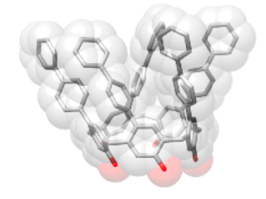

$(p-\mathrm{BiPh})_{5}(\mathrm{OH})_{5}-\mathrm{P}[5]$

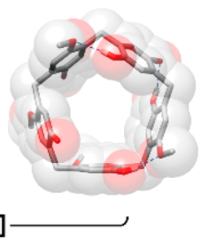

(d)

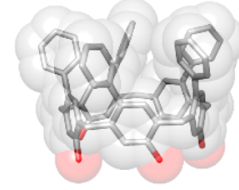

$(\mathrm{Ph})_{5}(\mathrm{OH})_{5}-\mathrm{P}[5]$

(g)

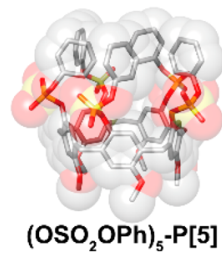

(b)

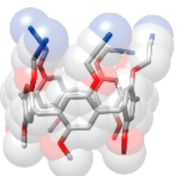

(e)
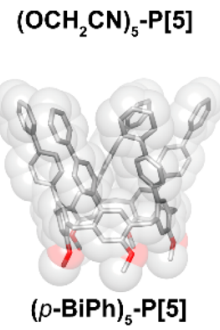

(h)

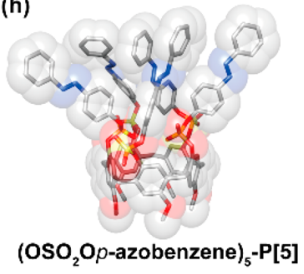

Figure 2. X-ray solid-state structures of representative compounds, depicted in a blend of tubular stick and space-filling representations: (a) side and top views of $(\mathbf{O H})_{5}-\mathbf{P}[5]$, (b) $\left(\mathrm{OCH}_{2} \mathbf{C N}\right)_{5}-\mathbf{P}[\mathbf{5}]$, (c) $(\mathrm{Ph})_{5} \mathrm{P}[5],(\mathrm{d})(\mathrm{Ph})_{5}(\mathrm{OH})_{5}-\mathrm{P}[5],(\mathrm{e})(p-\mathrm{BiPh})_{5}-\mathrm{P}[5]$, (f) (p$\mathrm{BiPh}_{5}(\mathrm{OH})_{5}-\mathrm{P}[5](\mathrm{g})\left(\mathrm{OSO}_{2} \mathrm{OPh}\right)_{5}-\mathrm{P}[5]$, (h) (OSO $\mathrm{O}$-azobenzene $)_{5}-\mathbf{P}[\mathbf{5}]$. All these compounds crystallize as racemates, and only one enantiomeric conformer is shown. Hydrogen atoms and solvents are omitted for clarity. Color code: carbon, gray; oxygen, red; nitrogen, blue, sulfur, yellow.

In summary, a series of new $C_{5}$-symmetric macrocycles derived from the pillar[5] arene family was synthesized in good to excellent yields, starting from a common penta-hydroxy rimdifferentiated $\mathrm{P}[5]$ intermediate. Reaction of the $-\mathrm{OH}$ moieties via alkylation, esterification, or SuFEx strategies allows a broad variety of substituents to be installed. In addition, conversion of the $-\mathrm{OH}$ moieties to triflates, followed by Suzuki-Miyaura coupling affords removal of the $\mathrm{O}$ atoms and $\mathrm{C}-\mathrm{C}$ based structures, with altogether different steric and electronic properties. Finally, subsequent removal of the methyl groups on the lower rim opens this side up to analogous functionalization schemes, thus allowing pillar[5]arenes to be functionalized fully at will on both sides. This will further broaden the scope of the chemistry of 5-fold symmetric macrocycles via additional studies of host-guest interactions, substitution effects and solid-state properties, and likely yields wide application, especially via novel building blocks, in supramolecular architectures, reticular chemistry, and functional $\pi$-systems.

\section{ASSOCIATED CONTENT}

\section{S Supporting Information}

The Supporting Information is available free of charge on the ACS Publications website at DOI: 10.1021/acs.orglett.9b01123.

Synthesis procedures, yields, and characterization data $\left({ }^{1} \mathrm{H}\right.$ NMR, ${ }^{13} \mathrm{C}$ NMR, HR-MS, UV-vis) (PDF)

\section{Accession Codes}

CCDC 1896018, 1896019, 1896021,1896022,1896023, 1896026,1904736 , and 1904933 contain the supplementary crystallographic data for this paper. These data can be obtained free of charge via www.ccdc.cam.ac.uk/data request/cif, or by emailing data_request@ccdc.cam.ac.uk, or by contacting The Cambridge Crystallographic Data Centre, 12 Union Road, Cambridge CB2 1EZ, UK; fax: +44 1223336033.

\section{AUTHOR INFORMATION}

\section{Corresponding Authors}

*E-mail: andrew.sue@tju.edu.cn (A. C.-H. Sue).

*E-mail: han.zuilhof@wur.nl (H. Zuilhof).

\section{ORCID $\odot$}

Paul Demay-Drouhard: 0000-0003-2270-1177

Ke Du: 0000-0002-1786-7327

Kushal Samanta: 0000-0002-7414-4475

Xintong Wan: 0000-0002-5792-0030

Weiwei Yang: 0000-0003-3062-6023

Rajavel Srinivasan: 0000-0001-8374-1952

Andrew C.-H. Sue: 0000-0001-9557-2658

Han Zuilhof: 0000-0001-5773-8506

Author Contributions

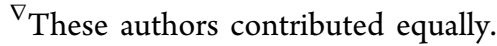

Notes

The authors declare no competing financial interest.

\section{ACKNOWLEDGMENTS}

This work was supported by the 973 National Basic Research Program of China (No. 2015CB856500, to A.C.-H.S. and H.Z.), National Science Foundation of China (No. 21871208, to H.Z.), National Thousand Young Talents Program of China (to A.C.-H.S.), the Tianjin City Thousand Talents Program (to A.C.-H.S. and H.Z.), and Wageningen University.

\section{REFERENCES}

(1) Steed, J. W.; Atwood, J. L. Supramolecular Chemistry, 2nd Edition; Wiley-VCH, 2009.

(2) Crini, G. Chem. Rev. 2014, 114, 10940-10975.

(3) Böhmer, V. Angew. Chem., Int. Ed. Engl. 1995, 34, 713-745.

(4) Gutsche, C. D. Calixarenes, An Introduction, 2nd Edition; RSC, 2008.

(5) Ogoshi, T.; Kanai, S.; Fujinami, S.; Yamagishi, T. A.; Nakamoto, Y. J. Am. Chem. Soc. 2008, 130, 5022-5023.

(6) Ogoshi, T.; Yamagishi, T. A.; Nakamoto, Y. Chem. Rev. 2016, 116, 7937-8002.

(7) Kakuta, T.; Yamagishi, T. A.; Ogoshi, T. Acc. Chem. Res. 2018, $51,1656-1666$

(8) Zhang, H.; Liu, Z.; Zhao, Y. Chem. Soc. Rev. 2018, 47, 54915528.

(9) Ogoshi, T.; Sueto, R.; Yoshikoshi, K.; Sakata, Y.; Akine, S.; Yamagishi, T. A. Angew. Chem., Int. Ed. 2015, 54, 9849-9852.

(10) Ogoshi, T.; Shimada, Y.; Sakata, Y.; Akine, S.; Yamagishi, T. A. J. Am. Chem. Soc. 2017, 139, 5664-5667.

(11) Ogoshi, T.; Demachi, K.; Kitajima, K.; Yamagishi, T. A. Chem. Commun. 2011, 47, 10290-10292.

(12) Jie, K.; Zhou, Y.; Li, E.; Li, Z.; Zhao, R.; Huang, F. J. Am. Chem. Soc. 2017, 139, 15320-15323.

(13) Jie, K.; Zhou, Y.; Yao, Y.; Shi, B.; Huang, F. J. Am. Chem. Soc. 2015, 137, 10472-10475.

(14) Jie, K.; Zhou, Y.; Li, E.; Huang, F. Acc. Chem. Res. 2018, 51, 2064-2072.

(15) Li, E.; Zhou, Y.; Zhao, R.; Jie, K.; Huang, F. Angew. Chem., Int. Ed. 2019, 58, 3981-3985.

(16) Ogoshi, T.; Akutsu, T.; Yamafuji, D.; Aoki, T.; Yamagishi, T. Angew. Chem., Int. Ed. 2013, 52, 8111-8115. 
(17) Song, N.; Kakuta, T.; Yamagishi, T.; Yang, Y.; Ogoshi, T. Chem. 2018, 4, 2029-2053.

(18) Wang, X.; Wang, W.; Li, W.; Chen, L.; Yao, R.; Yin, G.; Wang, Y.; Zhang, Y.; Huang, J.; Tan, H.; Yu, Y.; Li, X.; Xu, L.; Yang, H.-B.; et al. Nat. Commun. 2018, 9, 3190.

(19) Langer, P.; Yang, L.; Pfeiffer, C. R.; Lewis, W.; Champness, N. R. Dalt. Trans. 2019, 48, 58-64.

(20) Nierengarten, I.; Guerra, S.; Holler, M.; Karmazin-Brelot, L.; Barbera, J.; Deschenaux, R.; Nierengarten, J.-F. Eur. J. Org. Chem. 2013, 2013, 3675-3684.

(21) Vincent, S. P.; Buffet, K.; Nierengarten, I.; Imberty, A.; Nierengarten, J.-F. Chem. - Eur. J. 2016, 22, 88-92.

(22) Strutt, N. L.; Zhang, H.; Schneebeli, S. T.; Stoddart, J. F. Acc. Chem. Res. 2014, 47, 2631-2642.

(23) Ogoshi, T.; Demachi, K.; Kitajima, K.; Yamagishi, T. Chem. Commun. 2011, 47, 7164-7166.

(24) Strutt, N. L.; Forgan, R. S.; Spruell, J. M.; Botros, Y. Y.; Stoddart, J. F. J. Am. Chem. Soc. 2011, 133, 5668-5671.

(25) Zhang, Z.; Xia, B.; Han, C.; Yu, Y.; Huang, F. Org. Lett. 2010, $12,3285-3287$

(26) Strutt, N. L.; Fairen-Jimenez, D.; Iehl, J.; Lalonde, M. B.; Snurr, R. Q; Farha, O. K.; Hupp, J. T.; Stoddart, J. F. J. Am. Chem. Soc. 2012, 134, 17436-17439.

(27) Al-Azemi, T. F.; Mohamod, A. A.; Vinodh, M.; Alipour, F. H. Org. Chem. Front. 2018, 5, 10-18.

(28) Bojtár, M.; Simon, A.; Bombicz, P.; Bitter, I. Org. Lett. 2017, 19, $4528-4531$.

(29) Strutt, N. L.; Zhang, H.; Schneebeli, S. T.; Stoddart, J. F. Chem. - Eur. J. 2014, 20, 10996-11004.

(30) Fu, S.; An, G.; Sun, H.; Luo, Q.; Hou, C.; Xu, J.; Dong, Z.; Liu, J. Chem. Commun. 2017, 53, 9024-9027.

(31) Meichsner, E.; Nierengarten, I.; Holler, M.; Chessé, M.; Nierengarten, J.-F. Helv. Chim. Acta 2018, 101, No. e1800059.

(32) Kou, Y.; Tao, H.; Cao, D.; Fu, Z.; Schollmeyer, D.; Meier, H. Eur. J. Org. Chem. 2010, 2010, 6464-6470.

(33) Zhang, Z.; Luo, Y.; Xia, B.; Han, C.; Yu, Y.; Chen, X.; Huang, F. Chem. Commun. 2011, 47, 2417-2419.

(34) Yu, G.; Zhang, Z.; Han, C.; Xue, M.; Zhou, Q.; Huang, F. Chem. Commun. 2012, 48, 2958-2960.

(35) Shu, X.; Chen, W.; Hou, D.; Meng, Q.; Zheng, R.; Li, C. Chem. Commun. 2014, 50, 4820-4823.

(36) Zhou, Y.; Yao, Y.; Huang, F. Chin. J. Chem. 2015, 33, 356-360.

(37) Ding, J.; Chen, J.; Mao, W.; Huang, J.; Ma, D. Org. Biomol. Chem. 2017, 15, 7894-7897.

(38) Guo, M.; Wang, X.; Zhan, C.; Demay-Drouhard, P.; Li, W.; Du, K.; Olson, M. A.; Zuilhof, H.; Sue, A. C. H. J. Am. Chem. Soc. 2018, 140, 74-77.

(39) Yao, Y.; Xue, M.; Chen, J.; Zhang, M.; Huang, F. J. Am. Chem. Soc. 2012, 134, 15712-15715.

(40) Yu, G.; Ma, Y.; Han, C.; Yao, Y.; Tang, G.; Mao, Z.; Gao, C.; Huang, F. J. Am. Chem. Soc. 2013, 135, 10310-10313.

(41) Zhang, H.; Ma, X.; Nguyen, K. T.; Zhao, Y. ACS Nano 2013, 7, $7853-7863$.

(42) Nishimura, T.; Sanada, Y.; Matsuo, T.; Okobira, T.; Mylonas, E.; Yagi, N.; Sakurai, K. Chem. Commun. 2013, 49, 3052-3054.

(43) Yao, Y.; Xue, M.; Zhang, Z.; Zhang, M.; Wang, Y.; Huang, F. Chem. Sci. 2013, 4, 3667-3672.

(44) Zhou, J.; Chen, M.; Xie, J.; Diao, G. ACS Appl. Mater. Interfaces 2013, 5, 11218-11224.

(45) Luo, L.; Nie, G.; Tian, D.; Deng, H.; Jiang, L.; Li, H. Angew. Chem., Int. Ed. 2016, 55, 12713-12716.

(46) Al-Azemi, T. F.; Vinodh, M.; Alipour, F. H.; Mohamod, A. A. J. Org. Chem. 2017, 82, 10945-10952.

(47) Ogoshi, T.; Aoki, T.; Kitajima, K.; Fujinami, S.; Yamagishi, T. A.; Nakamoto, Y. J. Org. Chem. 2011, 76, 328-331.

(48) Dong, J.; Krasnova, L.; Finn, M. G.; Sharpless, K. B. Angew. Chem., Int. Ed. 2014, 53, 9430-9448.

(49) Gahtory, D.; Sen, R.; Pujari, S.; Li, S.; Zheng, Q.; Moses, J. E.; Sharpless, K. B.; Zuilhof, H. Chem. - Eur. J. 2018, 24, 10550-10556.
(50) Ogoshi, T.; Kida, K.; Yamagishi, T. J. Am. Chem. Soc. 2012, 134, 20146-20150.

(51) Ogoshi, T.; Takashima, S.; Yamagishi, T. J. Am. Chem. Soc. 2018, 140, 1544-1548.

(52) Bruno, N. C.; Tudge, M. T.; Buchwald, S. L. Chem. Sci. 2013, 4, 916-920.

(53) Ogoshi, T.; Umeda, K.; Yamagishi, T.; Nakamoto, Y. Chem. Commun. 2009, 4874-4876. 